\title{
Nueva técnica para control de contaminación de fístulas enteroatmosféricas en abdomen abierto Björck 4. Método de Capilaridad (MECA)
}

\author{
New technique to control enterocutaneous fistulas contamination in open \\ abdomen Björck 4. Capillarity method (MECA)
}

Edwin Oveimar Muñoz-Ruiz $\mathbb{D}^{\mathbb{D}}$, Natalia María Bravo-Flórez² $\mathbb{D}$, Jorge Augusto Herrera-Chaparro' ${ }^{\mathbb{D}}$, Guillermo Vallejo-Vallecilla ${ }^{3} \mathbb{D}$

\footnotetext{
1. Médico, especialista en Cirugía general, Clínica La Estancia, Popayán, Colombia. Clinical Epidemiology training, Karolinskia Institutet, Sweden. Fellow Gastroenterología Clínico quirúrgica, Universidad de Caldas, Colombia.

2. Médica, Unidad de Cuidados Intensivos, Clínica La Estancia, Popayán, Colombia.

3. Médico, especialista en Cirugía general y Cirugía de transplantes, Clínica La Estancia, Popayán, Colombia.
}

\section{Resumen}

Introducción. La fístula enteroatmosférica es una patología compleja que puede ser el resultado de múltiples intervenciones quirúrgicas de la cavidad abdominal. Describimos una nueva técnica para el control de la contaminación en pacientes con fístulas enteroatmosféricas en abdomen abierto Björck 4.

Métodos. Se realizó un análisis retrospectivo de cuatro pacientes con fístulas enteroatmosféricas desarrolladas como complicación de procedimientos quirúrgicos abdominales. Se trataron integralmente por un grupo multidisciplinario de Cuidados Intensivos, Cirugía general, Soporte nutricional y Cuidado de heridas y ostomías. Se utilizó una novedosa técnica quirúrgica basada en el principio de capilaridad para mantener limpios los tejidos periostomales.

Resultados. Con esta técnica se logró el control de la contaminación abdominal en todos los pacientes y una evolución clínica satisfactoria. Posteriormente se programaron para cierre quirúrgico definitivo con éxito.

Conclusiones. El manejo de la fístula enteroatmosférica representa un reto para el cirujano y el grupo multidisciplinario que trata al paciente. Esta nueva técnica utilizada en pacientes con fístula enteroatmosférica con abdomen abierto Björck 4 se basa en el principio de capilaridad, y es eficaz en el control de la contaminación, infección y de la sepsis asociada.

Palabras clave: fístula intestinal; capilar; peritonitis; sepsis; cirugía general.

Fecha de recibido: 21/05/2021 - Fecha de aceptación: 05/09/2021 - Publicación en línea: 25/10/2021

Autor de correspondencia. Edwin Oveimar Muñoz-Ruiz, Calle 34 Norte \# 13 N 43, edificio Atardeceres de Campobello, apartamento 505, Popayán, Colombia. Teléfono: 3178536620. Correo electrónico: edwinmunoz@unicauca.edu.co

Citar como: Muñoz-Ruiz EO, Bravo-Flórez NM, Herrera-Chaparro JA, Vallejo-Vallecilla G. Nueva técnica para control de contaminación de fístulas enteroatmosféricas en abdomen abierto Björck 4. Método de Capilaridad (MECA). Rev Colomb Cir. 2022;37:90-5. https://doi.org/10.30944/20117582.939

Este es un artículo de acceso abierto bajo una Licencia Creative Commons - BY-NC-ND https://creativecommons.org/licenses/by-nc-nd/4.0/deed.es 


\begin{abstract}
Introduction. Enterocutaneous fistula is a complex pathology that can be the result of multiple surgical interventions of the abdominal cavity. We describe a new technique for the control of contamination in patients with enterocutaneous fistulas in the open abdomen Björck 4.

Methods. A retrospective analysis of four patients with enterocutaneous fistulas developed as a complication of abdominal surgical procedures was performed. They were treated by a multidisciplinary team of Intensive Care, General Surgery, Nutritional support and Wound and ostomy care. A novel surgical technique based on the capillarity principle was used to keep the periostomies tissues clean.

Results. With this technique, control of abdominal contamination was achieved in all patients and a satisfactory clinical evolution. Later they were scheduled for definitive surgical closure with total success.

Conclusions. The management of an enterocutaneous fistula represents a challenge for the surgeon and the multidisciplinary group that treats these patients. This new technique used in patients with an enterocutaneous fistula with open abdomen Björck 4 is based on the principle of capillarity action, and is effective in controlling contamination, infection, and associated sepsis.
\end{abstract}

Keywords: intestinal fistula; capillary; peritonitis; sepsis; general surgery.

\section{Introducción}

La fístula enteroatmosférica (FEA) se define como una comunicación anormal entre la luz del tracto digestivo y la atmosfera. Es una patología compleja que puede ocurrir como complicación de múltiples y repetidas intervenciones quirúrgicas de la cavidad abdominal y conforma un subgrupo especial de las fístulas intestinales ${ }^{1}$. Esta condición patológica afecta el funcionamiento normal del tracto digestivo, condicionando una falla intestinal que altera la capacidad para mantener un estado nutricional adecuado ${ }^{2}$. Debido a su alta tasa de morbilidad y mortalidad con afectación de la calidad de vida del paciente, el tratamiento representa un gran reto para el cirujano e implica una estancia hospitalaria prolongada, especialmente en la Unidad de Cuidados Intensivos (UCI), mayor tiempo de soporte nutricional, aumento en el número de intervenciones quirúrgicas y, por lo tanto, en los costos de atención ${ }^{3-5}$.

Cerca del $80 \%$ de las fístulas enteroatmosféricas corresponden a una complicación de las cirugías abdominales y, a mayor número de reintervenciones, mayor riesgo de desarrollarlas ${ }^{1,6}$. El estado de nutrición del paciente juega un papel importante en el desarrollo de dichas complicaciones. En diferentes series se ha documentado que hasta un $40 \%$ de los pacientes operados tiene algún grado de desnutrición, lo que incrementa la probabilidad de presentar una fístula enteroatmosférica.

Uno de los mayores problemas en los pacientes que han desarrollado FEA es el control de la contaminación abdominal, que contribuye directamente a la sepsis asociada y al deterioro de su condición clínica con una alta tasa de mortalidad ${ }^{6,7}$.

El objetivo de este trabajo es dar a conocer una novedosa técnica denominada "método de capilaridad", que, de manera fácil, práctica y efectiva, permitió el control de la contaminación local en cuatro pacientes con fistulas entero atmosféricas, tratados de forma exitosa en nuestra institución.

\section{Métodos}

Entre los años 2017 y 2021 se identificaron de manera retrospectiva cuatro pacientes previamente sanos y con un buen estado nutricional, quienes como complicación de procedimientos quirúrgicos abdominales desarrollaron fistulas entero atmosféricas. Los pacientes presentaron inicialmente sepsis abdominal y fueron tratados con abdomen abierto por la necesidad de reintervenciones para el control de la peritonitis. Todos presentaron fístulas que fueron tratadas mediante 
una novedosa técnica basada en el método de capilaridad (MECA).

Adicionalmente recibieron una atención multidisciplinaria por parte de los servicios de Cirugía general, Cuidado crítico, Nutrición y Cuidado de heridas y ostomías. Se corrigieron los trastornos electrolíticos, se ofreció manejo antibiótico temprano, soporte nutricional y control de la contaminación abdominal con cirugía.

\section{Principio de capilaridad}

La capilaridad es una propiedad de los fluidos que depende de dos fuerzas, la cohesión y la adhesión. La cohesión es la atracción entre moléculas semejantes y la adhesión es una atracción entre moléculas distintas en contacto, como la del líquido a un tubo, lo cual le confiere la capacidad de subir a través de él ${ }^{8}$.

\section{Técnica de manejo de fistulas por principio de capilaridad}

Esta novedosa técnica nace de la idea de aplicar un principio físico (la capilaridad), usando la fuerza de tensión de un fluido (liquido intestinal) en un tubo, para dirigir el contenido intestinal de la fistula al exterior mediante un efecto de succión.

Se utiliza un tubo de látex de $15 \mathrm{~cm}$ de longitud perforado en el extremo proximal con orificios de $5 \mathrm{~mm}$, diámetro que puede variar de acuerdo con el tamaño de la fistula. El tubo se introduce $5 \mathrm{~cm}$ en la luz del intestino y posteriormente se sutura en los bordes de la fistula con material no absorbible de calibre 5-0 (Figura 1). El espacio periostomal a proteger se debe cubrir con una bolsa de viaflex (bolsa de Borráez) fijada a la piel ${ }^{9}$, brindando el mayor recubrimiento posible y se debe perforar para permitir el paso del tubo que emerge de la o de las fístulas. Este cubrimiento permite la granulación del tejido periostomal y lo aísla de la contaminación (Figura 2).

Sobre la bolsa plástica se debe instalar una barrea y una bolsa recolectora de colostomía como reservorio para la producción de la fístula (Figura 3). Una vez granulada el área alrededor de la fístula, la bolsa se debe sustituir por un

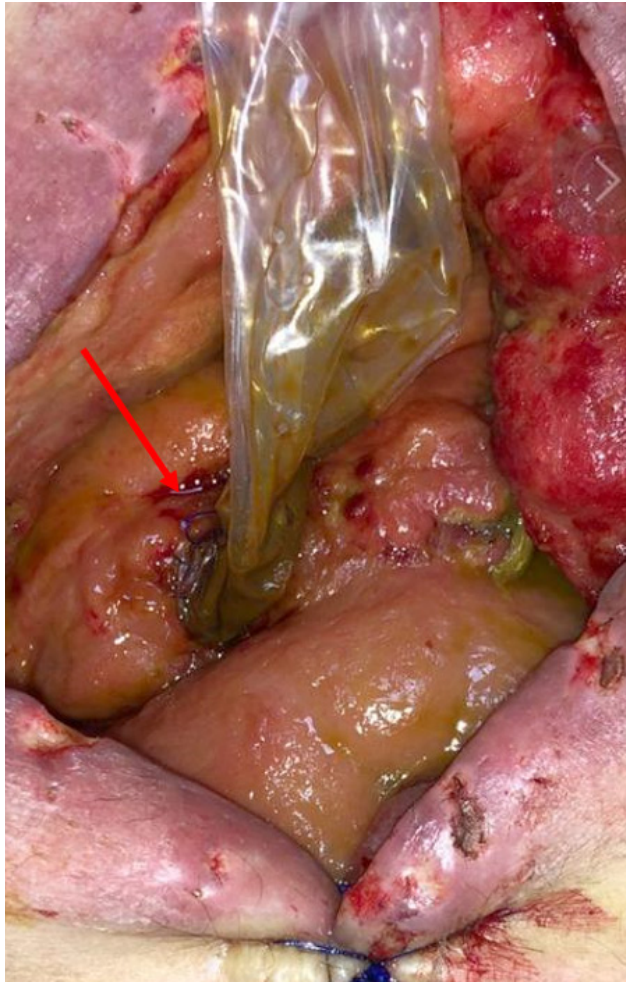

Figura 1. Técnica de manejo de fístulas por principio de capilaridad, la flecha indica el sitio de sutura del tubo de látex a la fístula de intestino delgado.

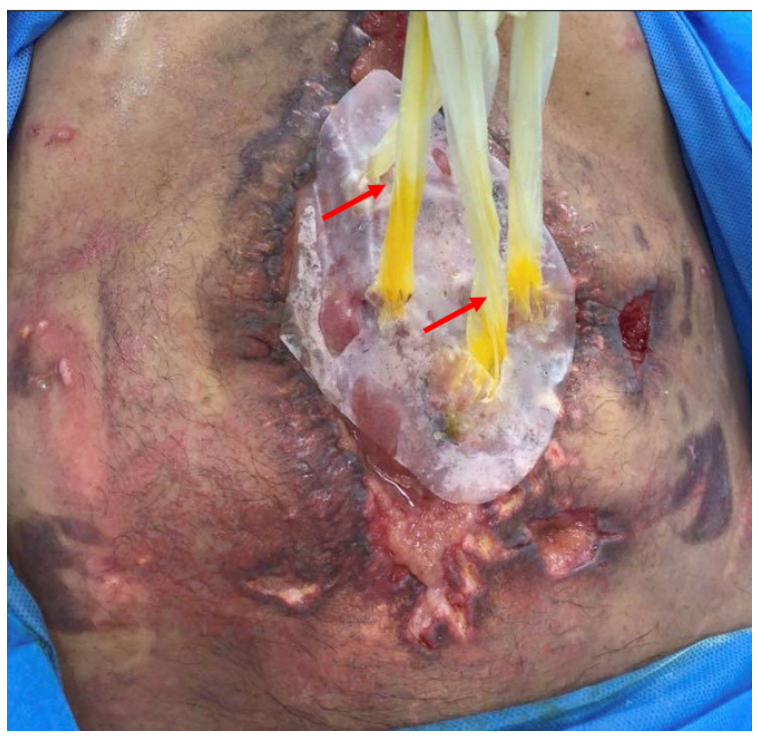

Figura 2. Técnica de manejo de fístulas por principio de capilaridad, las flechas indican el nivel de líquido que sube por el tubo de látex. 


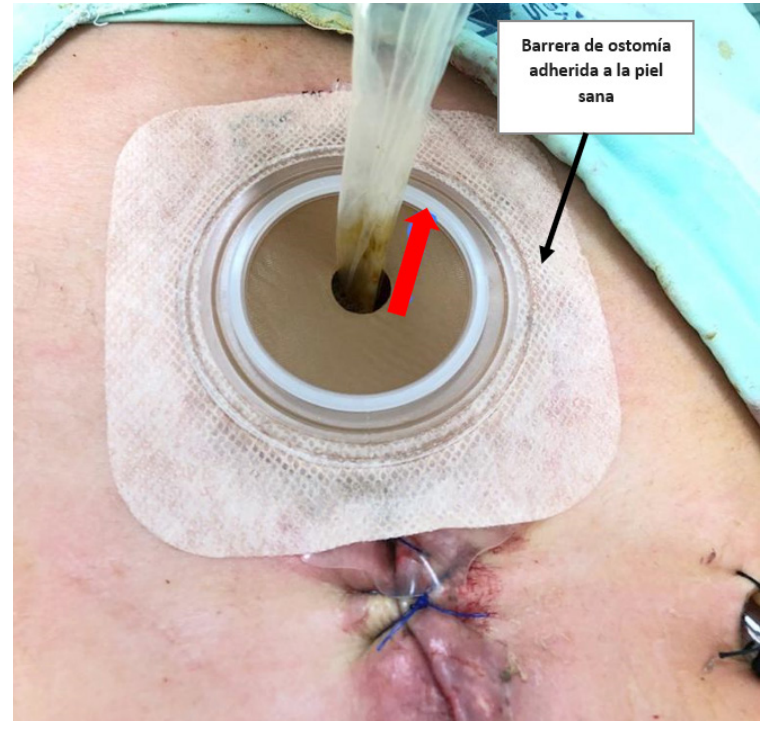

Figura 3. Técnica de manejo de fístulas por principio de capilaridad. La flecha roja indica la dirección del nivel de líquido que sube por el tubo de látex el cual emerge por la barrera de ostomía protegiendo la piel y el tejido periostomal.

apósito especial de hidrocoloide y desde ambos lados de la herida abdominal se deben tallar colgajos cutáneos para aproximarlos a la línea media, buscando el mayor cubrimiento posible de la cavidad abdominal con cada nueva revisión quirúrgica que se realice. Este método controla la contaminación periostomal y disminuye la carga séptica del paciente, favoreciendo la granulación del tejido y disminuyendo el número de reintervenciones.

\section{Resultados}

En este trabajo se incluyeron cuatro pacientes cuyas características se presentan en la tabla 1. Todos fueron hombres, con edad promedio de 46 años. La indicación de la primera cirugía fue obstrucción intestinal en dos pacientes con antecedente de trauma abdominal, cierre de ileostomía también con antecedente de trauma abdominal en otro paciente y una cirugía de urgencias por sepsis abdominal con diagnóstico de apendicitis aguda en el último paciente.

Los dos pacientes intervenidos por obstrucción intestinal y el de sepsis abdominal, fueron tratados desde un comienzo con abdomen abierto y desarrollaron fístulas debido a la liberación de adherencias y a fuga del muñón apendicular respectivamente. El paciente llevado a cierre de ileostomía, presentó fuga de la anastomosis por lo que debió ser reintervenido y dejado en abdomen abierto.

Todos los pacientes presentaron abdomen abierto Björck 4 y el número de intervenciones promedio fue de 7,2 cirugías por caso. Una vez se inició el manejo con la técnica de capilaridad, todos los pacientes mejoraron su estado de sepsis, requirieron menos procedimientos quirúrgicos para lavado de cavidad abdominal y pasaron más rápido a manejo por el grupo de heridas y ostomías. Los cuatro pacientes se programaron para cierre quirúrgico de la pared abdominal con éxito y egresaron después de un promedio de 55 días de estancia en hospitalización, con seguimiento posquirúrgico ambulatorio satisfactorio.

Tabla 1. Características clínicas y demográficas de los pacientes estudiados

\begin{tabular}{|c|c|c|c|c|c|c|}
\hline Paciente & $\begin{array}{l}\text { Edad } \\
\text { (años) }\end{array}$ & Genero & Causa de FEA & $\begin{array}{l}\text { Número de } \\
\text { fístulas }\end{array}$ & $\begin{array}{l}\text { Número de } \\
\text { cirugías }\end{array}$ & $\begin{array}{c}\text { Días de } \\
\text { hospitalización }\end{array}$ \\
\hline 1 & 39 & Masculino & $\begin{array}{l}\text { Liberación de } \\
\text { adherencias }\end{array}$ & 18 & 12 & 126 \\
\hline 2 & 75 & Masculino & $\begin{array}{l}\text { Fuga del muñón } \\
\text { apendicular }\end{array}$ & 3 & 5 & 30 \\
\hline 3 & 45 & Masculino & $\begin{array}{l}\text { Liberación de } \\
\text { adherencias }\end{array}$ & 3 & 7 & 40 \\
\hline 4 & 25 & Masculino & $\begin{array}{c}\text { Fuga de anastomosis } \\
\text { íleo-colónica }\end{array}$ & 1 & 5 & 24 \\
\hline
\end{tabular}

FEA: fístula entero atmosférica. 


\section{Discusión}

La fístula enteroatmosférica en un abdomen abierto Björck 4 es de difícil manejo y existen diversas maneras de abordar estos pacientes. Uno de los métodos empleados más comúnmente es la bolsa de Borráez o de Bogotá, con un catéter, sonda u otro dispositivo para recoger el efluente desde el lumen intestinal ${ }^{9-11}$. Otra opción que ha venido en auge es la utilización sobre el abdomen abierto del sistema de cierre por presión negativa también conocido como sistema VAC (Vacuum Assisted Closure), con el fin de controlar la contaminación local.

Este último método se ha combinado con otras técnicas como la FISPME (fistula isolation by suturing the Penrose drain to mucosa of orifice of entero-atmospheric fistula) descrita por Yetisir et al., en la cual se utilizó un solo dren de Penrose corto suturado al borde de varias fístulas en conjunto para aislar su producción, mientras que el área periostomal se protegió con sistema VAC ${ }^{12}$. Eğin et al., modificaron esta técnica y la denominaron NITS (new isolation technique with stopper), en la cual, luego de suturar un dren de Penrose corto al lumen intestinal, se ocluye la fístula con un apósito hidrocoloide y se utiliza un VAC en el área periostomal ${ }^{13}$. En ambos trabajos se lograron desenlaces favorables.

En un trabajo publicado en 2017, Rivera presentó su técnica que consiste en suturar la parte proximal del intestino a un preservativo, con lo que se logra dirigir la producción de la fistula hacia una bolsa de colostomía aislando la piel, sin embargo, este procedimiento permite cierto grado de fuga y el látex tiende a romperse rápidamente ${ }^{14}$. En la técnica que presentamos, la sutura del dren al borde de la fístula genera mayor hermetismo y más fuerza de capilaridad, permitiendo orientar el fluido al exterior con escasa o nula fuga por los bordes, y disminuyendo el número de reintervenciones en el paciente.

En la serie de pacientes presentada por Wang et al., el promedio de días para el cierre de las fistulas fue de $128,1^{15}$, similar al tiempo de nuestro primer paciente, pero teniendo en cuenta que el promedio general de nuestros pacientes fue menor. No hubo mortalidad en nuestro grupo de pacientes, acorde con la mayoría de trabajos publicados ${ }^{10,16,17}$.

\section{Conclusión}

La técnica MECA permite aislar la contaminación hacia una bolsa recolectora, con un notable impacto en la mejoría de los pacientes, sobre todo en la etapa de sepsis, en la que es más difícil el control de la contaminación periostomal.

Esta técnica no afecta la seguridad del paciente, no requiere un alto grado de entrenamiento, es de fácil reproducción, y al no usar el sistema de vacío, disminuye los costos de manera importante, por lo que se propone su empleo para el difícil manejo de los pacientes con fístulas intestinales. Se considera importante el diseño y desarrollo de estudios adicionales con mejor diseño metodológico.

\section{Cumplimiento de normas éticas}

Consentimiento informado: Este trabajo se ciñe a las normas nacionales y según la resolución 008439 se considera de bajo riesgo para los pacientes, quienes dieron su consentimiento firmado para la publicación de este trabajo.

Conflicto de interés: Ninguno declarado por los autores.

Fuente de financiación: Financiado con recursos propios de los autores.

\section{Contribución de los autores:}

Concepción y diseño del estudio: Edwin Oveimar Muñoz-Ruiz, Natalia María Bravo-Flórez, Jorge Augusto Herrera-Chaparro, Guillermo Vallejo-Vallecilla.

Adquisición de datos: Edwin Oveimar Muñoz-Ruiz, Natalia María Bravo-Flórez, Jorge Augusto Herrera-Chaparro, Guillermo Vallejo-Vallecilla.

Análisis e interpretación de datos: Edwin Oveimar Muñoz-Ruiz, Natalia María Bravo-Flórez, Jorge Augusto Herrera-Chaparro, Guillermo Vallejo-Vallecilla.

Redacción del manuscrito: Edwin Oveimar Muñoz-Ruiz, Natalia María Bravo-Flórez, Jorge Augusto HerreraChaparro, Guillermo Vallejo-Vallecilla.

Revisión crítica: Edwin Oveimar Muñoz-Ruiz, Natalia María Bravo-Flórez, Jorge Augusto Herrera-Chaparro, Guillermo Vallejo-Vallecilla. 


\section{Referencias}

1. De Vries FE, Atema JJ, Van-Ruler O, Vaizey CJ, Serlie MJ, Boermeester MA. A systematic review and meta-analysis of timing and outcome of intestinal failure surgery in patients with enteric fistula. World J Surg. 2018;42:695-706.

https://doi.org/10.1007/s00268-017-4224-z

2. Grainger JT, Maeda Y, Donnelly SC, Vaizey CJ. Assessment and management of patients with intestinal failure: a multidisciplinary approach. Clin Exp Gastroenterol. 2018;11:233-41. https://doi.org/10.2147/CEG.S122868

3. Dudrick SJ, Maharaj AR, McKelvey AA. Artificial nutritional support in patients with gastrointestinal fistulas. World J Surg. 1999;23:570-6. https://doi.org/10.1007/PL00012349

4. Teixeira PGR, Inaba K, Dubose J, Salim A, Brown C, Rhee P, et al. Enterocutaneous fistula complicating trauma laparotomy: a major resource burden. Am Surg. 2009;75:302. https://doi.org/10.1177/000313480907500106

5. Wercka J, Cagol PP, Melo ALP, Locks G de F, Franzon O, Kruel NF, et al. Epidemiology and outcome of patients with postoperative abdominal fistula. Rev Colégio Bras Cir. 2016;43:117-23. https://doi.org/10.1590/0100-69912016002008

6. Torres OJM, Salazar RM, Costa JVG, Corrêa FCF, Malafaia O. Fístulas enterocutâneas pós-operatórias: análise de 39 pacientes. Rev Colégio Bras Cir 2002;29359-63.

7. Fuentes-Valdés E. Fístulas gastroenterocutáneas posoperatorias: factores que influyen en la mortalidad. Rev Cuba Cir. 2002;41:88-92. .

8. Castañeda A. Química. 11a Edición. Raymond Chang. Mc Graw Hill. Disponible en: https://www.academia. edu/36501670/Quimica_11va_Edicion_Raymond_ Chang_FREEL

9. Borráez OA. Abdomen abierto: la herida más desafiante. Rev Colomb Cir. 2008;23:204-9.
10. Borráez OA, Borráez BA. Cierre de heridas y fístulas con "sistema de presión negativa tipo Colombia". Rev Colomb Cir. 2009;24:236-43.

11. Klek S, Forbes A, Gabe S, Holst M, Wanten G, Irtun $\emptyset$, et al. Management of acute intestinal failure: A position paper from the European Society for Clinical Nutrition and Metabolism (ESPEN) Special Interest Group. Clin Nutr. 2016;35:1209-18. https://doi.org/10.1016/j.clnu.2016.04.009

12. Yetisir F, Sarer AE, Aldan M. New isolation technique for enteroatmospheric fistula in Björck 4 open abdomen. Hernia. 2017;21:809-12.

13. Eğin S, Gökçek B, Yeșiltaş M, Sağlam F, Güney B. Management of enteroatmospheric fistula thanks to new isolation technique. Ulus Travma Acil Cerrahi Derg. 2019;25:80-2. https://doi.org/10.5505/tjtes.2018.45267

14. Rivera MA. Quezada BK, Quiñónez M, Almada RR. Manejo de estomas complicados y/o abdomen hostil con la técnica de condón de Rivera. Diez años de experiencia. Cir Gen. 2017;39:82-92.

15. Wang G, Ren J, Liu S, Wu X, Gu G, Li J. "Fistula patch": Making the treatment of enteroatmospheric fistulae in the open abdomen easier. J Trauma Acute Care. Surg 2013;74:1175-7. https://doi.org/10.1097/TA.0b013e318282705e

16. Cuendis-Velázquez A, Trejo-Avila M, Arce-Liévano E, Cárdenas-Lailson E, Sanjuan-Martínez C, Moreno-Portillo M. A four-step technique for effluent diversion of enteroatmospheric fistulas. Wounds. 2019;31:285-91. Disponible en: https://pubmed.ncbi.nlm.nih.gov/31730510/

17. Bobkiewicz A, Walczak D, Smoliński S, Kasprzyk T, Studniarek A, Borejsza-Wysocki M, et al. Management of enteroatmospheric fistula with negative pressure wound therapy in open abdomen treatment: a multicentre observational study. Int Wound J. 2017;14:25564. https://doi.org/10.1111/iwj.12597 